\title{
A Local Causal Model of Spacetime Dynamics
}

\author{
Hans H. Diel \\ Diel Software Beratung und Entwicklung, Sindelfingen, Germany \\ Email: diel@netic.de
}

How to cite this paper: Diel, H.H. (2018) A Local Causal Model of Spacetime Dynamics. Open Access Library Journal, 5: e4957.

https://doi.org/10.4236/oalib.1104957

Received: October 3, 2018

Accepted: October 28, 2018

Published: October 31, 2018

Copyright $\odot 2018$ by author and Open Access Library Inc.

This work is licensed under the Creative Commons Attribution International License (CC BY 4.0).

http://creativecommons.org/licenses/by/4.0/

\begin{abstract}
The model of spacetime dynamics proposed here specifies how spacetime emerges, how changes of spacetime propagate, and how changes in spacetime arising from multiple sources accumulate. The overall goal of the model was to achieve maximum compatibility with general relativity and with Einstein's equation; this goal appears to be achievable except at very small scales, where the discreteness of the units of space matters. The elementary structure of space--time) that is assumed in this model is a derivative of causal dynamical triangulation. At the elementary level, space consists of a (discrete) number of interconnected space points, each of which is connected to a small number of neighbouring space points. The curvature of spacetime is expressed by the density of these space points and by the arrangement of the connections between them. Dynamics of spacetime (i.e., the emergence of space and the propagation of space changes) dynamically assigns "in-connections" and "out-connections" to the affected space points. Based on the model of the dynamics of curved discrete spacetime, a model of quantum field theory in curved discrete spacetime is described. Emergence and propagation of quantum fields (including particles) are mapped to the emergence and propagation of space changes by utilizing identical paths of in/out space point connections. Compatibility with standard quantum field theory requests the adjustment of the QFT techniques (e.g., Feynman diagrams, Feynman rules, creation/annihilation operators), which typically apply to three in/out connections, to $n>3$ in/out connections.
\end{abstract}

\section{Subject Areas \\ Quantum Mechanics, Theoretical Physics}

\section{Keywords}

Spacetime Models, Discrete Spacetime, Relativity Theory, Causal Models

\section{Introduction}

The author's attempt to construct a local causal model of quantum theory (QT), 
including quantum field theory (QFT), soon resulted in the recognition that a causal model of the dynamics of QT/QFT should better be based on a causal model of the dynamics of spacetime. Thus, a causal model of the dynamics of spacetime has been developed with the major goals (1) as much as possible compatibility with general relativity theory (GRT), and (2) the model should match the main features of the evolving model of QT/QFT, which are

- the model has to be a causal model,

- if possible, the model should be a local causal model,

- discreteness of the basic parameters (time, space, propagation paths).

The construction of a causal model of spacetime dynamics started with the search for some existing theory or model which might be at least a starting point for the model to be developed. Causal dynamical triangulation (CDT, see [1], [2], [3]) and more abstractly the concepts of loop quantum gravity (see [4] and [5]) were identified to match the author's requirements and thinking. The further model construction showed that, in order to come up with a local causal model according to the definitions given in [6] adaptations and refinements of the original CDT-based model appear appropriate. The adaptations and refinements concern basic GRT concepts such as 1) the elementary structure of space(-time), 2) the representation of space(-time) curvature, and 3) the relation between space and time. With GRT and special relativity theory (SRT), space and time are said to be integrated into spacetime. For the GRT-compatible model of spacetime dynamics, the integration of space and time remains, but with a different interpretation. The interpretation given in Section 3 has significant implications for the overall model of spacetime dynamics and the processes and algorithms described in Section 4.

The major goal for the development of a causal model of spacetime dynamics (Sections 2, 3 and 4) was to develop a model of the spacetime elementary structure that constitutes a suitable base for both the causal model of spacetime dynamics and the causal model of QT/QFT. The proposed model satisfies this goal. The emergence and propagation of quantum fields (including particles) is mapped to the emergence and propagation of space changes by utilizing identical paths of in/out-connections between space points. In Section 5, this major subject of the article is described.

In Section 6, application of the model of spacetime dynamics to cosmological dynamics is described.

\section{The Overall Model of Spacetime Dynamics}

The model of spacetime dynamics specifies these dynamics (i.e. the processes involved in the emergence of space and the changes in spacetime) in the form of a causal model. Causal models as defined in [6] demand a well-defined content and structure of these specifications and adherence to completeness and consistency rules. This enforces a maximum degree of completeness and consistency of the model. According to the definition of a causal model given in [6], a causal 
model consists of: 1) the specification of the system state (i.e. the complete set of objects and parameters that constitute the state of the physical system); and 2) a set of transition rules that specify how the system state changes depending on the relevant conditions. For the subject of this article (spacetime), the major component of the system state is space.

Definition 1. System state:=

space;

quantum objects;

Quantum objects represent the contents of the space ${ }^{1}$.

Time, the second component of "spacetime", is not an explicit part of the system state, but is an implicit constituent of the causal model. The causal model defined in [6] assumes continuous updating of the system state (i.e. the application of the state transition rules) at a constant time interval called the state update time interval (suti).

System state progression (state $S, \Delta t=$ suti) $:=\{$

DO UNTIL(nonContinueState $(S))\{$

$S \leftarrow$ applyLawsOfPhysics $(S, \Delta t) ;$

\}

\}

The separation of time from the system state appears to be a disintegration of space and time, contrary to the teachings of special relativity theory (SRT) and general relativity theory (GRT). This seeming disconnection of space and time is comparable to the treatment of time with the Hamiltonian formulation of general relativity (see, for example, [7]). In the model described here, the reintegration of space and time to achieve compatibility with GRT is obtained through the concept presented in Section 3.1.

\section{The Elementary Structure of Spacetime}

The elementary structure of spacetime that is assumed for the model described here has been derived from causal dynamical triangulation (CDT) (see [1], [2] and [3]). However, some adaptations were made to allow a better match to the requirements of a causal model:

1) As described above, time is not part of the system state in the causal model, and a time component therefore does not form part of the elementary units of space-time.

2) Unlike CDT, where the elementary units of spacetime are n-dimensional simplexes (e.g. triangles and tetrahedrons), in the model of spacetime dynamics, space simply consists of a set of interconnected space points. The question of whether these space points and their connections represent specific n-dimensional compounds, such as tetrahedrons for example, is left open and is not relevant to the model described here.

Definition 2. Space $:=\{$ spacepoint... $\}$;

${ }^{1}$ See Section 5 or [8] for more details on quantum objects. 


$$
\begin{aligned}
& \text { spacepoint }:=\{\psi, \text { gravitation spec, connections }\} \\
& \text { connections }:=\left\{\text { connection }_{1}, \ldots, \text { connection } n_{n}\right\} ; \\
& \text { connection }:=\{\text { neighbor spacepoint }, \text { direction }\}
\end{aligned}
$$

\subsection{The Space-Time Relationship}

In GRT and SRT, space and time are said to be integrated into spacetime. From a mathematical perspective, the integration of space and time is reflected in the use of vectors, matrices and tensors that combine the dimensions of space with that of time. The integration is also reflected in the laws of physics, where space and time (and their derivatives) are jointly transformed. As described above, in the causal model chosen here, space and time are strictly separated. Since this model also aims for maximal compatibility with GRT, the question arises of how this compatibility can be achieved with a model in which space and time are fundamentally (initially) not integrated. In the concept underlying the causal model of spacetime dynamics, spacetime integration does not apply to space and time in general, as in SRT and GRT; instead,

space-time integration only applies to physical processes executed in space and time.

This implies the following:

Assertion 1. The measure and metric for space and time can only be defined jointly for both space and time, and only with reference to a specific process that produces a specific rate of spatial change (i.e. length) within a specific time interval.

The physical process that is best suited for this joint definition of the measure for space and time is the movement of light, under the assumption that the speed of light is a constant.

Assertion 2. The execution speed of physical processes in terms of changes in length in relation to the execution time is invariant.

For example, if a clock rate (i.e. the proper time) changes, this is always accompanied by a length dilation in the space where the process is executed.

The major physical expressions of curved spacetime are length dilations and time dilations ${ }^{2}$ predicted (and observed) with GRT. "Time dilation" essentially means a dilation of the speed by which physical processes, such as clocks, run.

Assertion 3. As a special case of Assertions 1 and 2, length and time dilations are interrelated and occur only in combination.

Most physicists would probably consider Assertion 1 as being in line with GRT and SRT. Assertions 2 and 3, however, are probably not part of the generally agreed upon interpretation of GRT and SRT. It is unclear to what extent Assertions 2 and 3 deviate from the standard interpretation of SRT/GRT (see Section 7). Nevertheless, Assertions 2 and 3 are essential in the more detailed model of spacetime dynamics described below.

The above basic assertions with respect to the spacetime relationship lead to the following propositions concerning the elementary structure of spacetime:

${ }^{2}$ Throughout this article the term "dilation" is used to mean positive or negative dilation. 
Proposition 4. The state update time interval, suti is a constant of nature.

Proposition 5. The distance between two neighbouring space points, $I_{\text {connection }}$, is a constant of nature. This is the distance through which light moves during a state update time interval, suti.

(In Euclidean geometry, it is difficult to imagine that all space point connections have the same length if the connections are not restricted to orthogonal directions.)

In a model that assumes a constant speed of light, $c$, it follows from Propositions 4 and 5 that:

Proposition 6. During a state update time interval, suti, light moves a constant distance, namely the distance

$$
l_{\text {suti }}=l_{\text {connection }}=\operatorname{suti} \cdot \mathrm{c}
$$

The proposed model of spacetime dynamics assumes that all distances and lengths in space are composed of the elementary length units $I_{\text {suti }}$ Likewise, all time intervals are multiples of suti. Lengths and distances are defined only between two space points and only with reference to the speed of light $c$.

Proposition 7. The distance between two space points $s p_{1}$ and $s p_{2}$ is given by the number of spacepoints nsp $\left(s p_{1}, s p_{2}\right)$ through which light passes when moving from $s p_{1}$ to $s p_{2}$ multiplied by the elementary length unit $l_{\text {suti }}\left(=l_{\text {connection }}\right)$. distance $\left(s p_{1}, s p_{2}\right)=n s p\left(s p_{1}, s p_{2}\right) \cdot l_{\text {suti }}$.

The above propositions result in a model of spacetime in which the speed of light is a constant. However, due to Proposition 5, it is hard to avoid curved space. This does not present a problem, since curved spacetime is not undesirable in a spacetime model aiming for compatibility with GRT. The remaining problem is that of how to achieve GRT-compatible space curvature. Spacetime curvature due to time dilation (as predicted by GRT) also needs to be supported. The solution offered by Assertion 3 and Propositions 4-7 is that: 1) the process of space emergence/expansion (Section 4) results in length dilations through the suitable arrangement of space points; and that 2) length dilations cause clock rate dilations for processes running at space positions with dilated lengths.

The formal expression of point (1) is:

Proposition 8. Lengths within the gravitational field are dilated by the factor $F_{1}$.

The precise equation for the factor $F_{1}$ such that it is in accordance with GRT is given in Section 3.2 below. For the model described in this article, the revised formulation of Proposition 8 is:

Proposition 9. Physical processes run faster or slower depending on the length dilation at the position in which the respective physical process is executed.

Proposition 9 may be viewed as a refinement of Assertion 3 above. As in Assertion 3, the dilation of the clock rate concerns physical processes rather than the structure of spacetime. The major process that demonstrates the fixed relationship between the length dilation and the rate of change of the process is the 
propagation of light. This (simple) process is used as a measure for the change rate of other processes by setting the speed of light to be a constant, $c$. The next class of processes in which the rate of change depends on the length dilation in precisely the same proportions as in the propagation of light are clocks in differing realisations. In summary, there is no direct reflection of time dilation as an attribute of spacetime in the model of spacetime dynamics. Clock rate dilation (rather than time dilation) arises as a property of processes running within space. The clock rate dilation factor can be derived from the length dilation factor $F_{1}$ of the space points at which the respective process is currently being executed. Thus, in the model of spacetime dynamics, two levels of time are distinguished, although these are seen as a single entity in GRT/SRT:

1) At the basic level, the progression of time is determined by the uniform state update time interval, suti. Simultaneity is assumed for all state changes occurring within the same state update cycle.

2) Differing clock rates, proper times, and the relativity of simultaneity are not associated with the basic overall spacetime (level 1), but instead are associated with physical processes running in space.

In terms of space, two levels can also be distinguished, although these are two levels of consideration:

- At the abstract level (the mathematical level), the space consists of a set of interconnected space points. The issue of whether or not the totality of the interconnected space points represents a Euclidean space or a specific topology (e.g. a Riemann manifold) is left open.

- At the physical level (the essential level), physical meaning is assigned to the components of the space point and the space point connections. In particular, the length of the connections is no longer a geometrical property, but specifies only the $\Delta$ length through which light moves during the state update time interval, suti.

Thus, the integration of space and time into spacetime is established in the model of spacetime dynamics by the physical meaning assigned to the components of the space points and their connections.

\subsection{The Length Dilation Factor $F_{1}$}

In GRT, the curvature specification (i.e. the curvature tensor), contains a time-related component in addition to the three space-related components. As an example of the impact of the time factor, the gravitational redshift is explained as the consequence of the time factor in the spacetime curvature (see, for example, [9], page 231).

$$
\Delta s^{2}=-\left(1-\frac{2 G M}{c^{2} r}\right)(c \Delta t)^{2}+(\Delta x)^{2}+(\Delta y)^{2}+(\Delta z)^{2}
$$

This means that a clock at position $(x, y, z)$ would run slower than a clock that is not affected by a gravitational field by a factor 


$$
F_{1}=\sqrt{1-\frac{2 G M}{c^{2} r}}
$$

A standard clock at some point A of low potential (for example, at the surface of the earth) would run slower than the same clock at a point B with higher potential (for example, in a GPS satellite). Proposition 8 states that not only are the clock rates of clocks within a gravitational field dilated by the factor $F_{1}$, but that this dilation also applies to lengths. (As a supporting argument, only in this way can the assertion of the constant speed of light be maintained.) Proposition 9 also means that length dilation is the primary effect, and that the clock rate dilation for clocks residing in the length-dilated space is a consequence of the length dilation.

\section{The Spacetime Dynamics}

The overall specification of the spacetime dynamics is given by Einsteins equation

$$
G^{\alpha \beta}=\frac{8 \pi G}{c^{4}} T^{\alpha \beta} .
$$

A more detailed specification with reference to the elementary spacetime structure, the space points and the space point connections needs to be provided in terms of processes and algorithms that adhere to Einsteins equation as far as possible. In the description given in this article, three types of processes are distinguished: 1) the emergence of space from a single source; 2) changes in space curvature caused by a single source; and 3) the accumulation of space changes caused by multiple sources.

For a description of the processes of spacetime dynamics, it is necessary to first consider in more detail the elementary structure of spacetime with respect to the distribution of the space point connections and with regard to the representation of the gravitational potential. The gravitational potential is part of the gravitationspec contained in Definition 2 of Section 3.

Definition 3. gravitationspec $:=$ \{emergence-state, emergence-direction, gravitation-strength\};

The emergence state specifies whether the space point is the source of further space emergence within the next state update cycle. In the algorithm presented below, this is expressed as emergence-state = OUTPOINT. The emergence direction specifies the direction in which an "out" connection and a new space point primarily emerges. Whether further "out"-connections and further space points emerge depends on the gravitation strength and on the algorithm for the emergence of space changes. Figure 1 shows an example of a space point with 14 space point connections. Some of the connections are labelled in-1, in-2, ..., out-1, out- $2, \ldots$ (meaning inward connection 1 , inward connection $2, \ldots$, outward connection 1, outward connection 2 etc.). The association of inward and outward connections for a specific space point is a dynamical attribution. The outward connections at state update cycle $\mathrm{i}$ are the inward connections at 


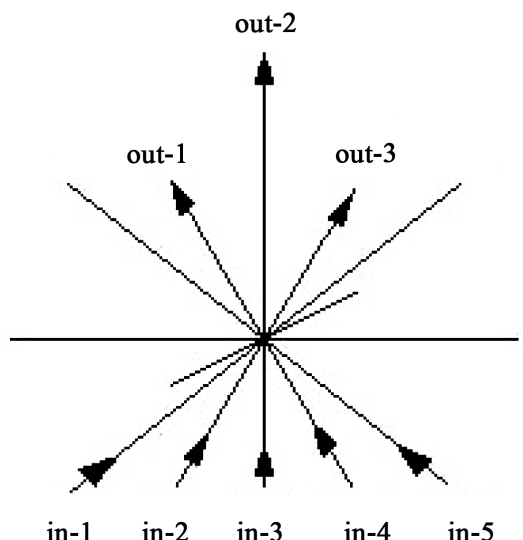

Figure 1. The distribution of connections of a space point.

the next state update cycle $(i+1)$. The attribution applies only to those space points which are currently involved in the emergence of space changes (i.e. emergence-state $=$ OUTPOINT). The determination of which space point connections are outward connections (and thus inward connections to the next state update cycle) depends on the distribution of the inward connections and on the more detailed space emergence algorithm.

The general algorithm for the propagation of space changes is embedded in the overall causal model of space dynamics. The space-state progression algorithm consists of the repeated application of split/join operations to all space points that are identified as OUTPOINTs (i.e., emergence-state $=$ OUTPOINT).

Specification 4. Space-state-progression (space $s p):=\{$

Split. FOR (ALL spacepoints sp.point $[i])\{$

IF (sp.point $[i]$.emergence-state $=$ OUTPOINT $)$

generate-new-points-from(sp.point $[i])$;

\}

Join: FOR (ALL spacepoints sp.point $[j])\{$

$I F($ sp.point $[j]=I N P O I N T)$ accumulate-inconnections $($ sp.point $[j])$;

\}

\}

The expression "generate-new-points-from( )" generates new space points (including the necessary connections) for all space points that are currently labelled sp.emergence-state $=$ OUTPOINT. At least one new space point is generated. Whether further outward connections and further space points emerge depends on the gravitation strength and on the more detailed algorithm for the emergence of space changes. If multiple space points and outward connections are generated, the gravitation strength is distributed among the multiple new space points, after it is reduced as a function of the increasing distance from the gravitational source(s). The newly generated space points are temporarily marked as INPOINTs for the next step of the process, in which the gravitational strengths are accumulated for all connections of a given INPOINT. The overall algorithm can be expressed by the following specification. 


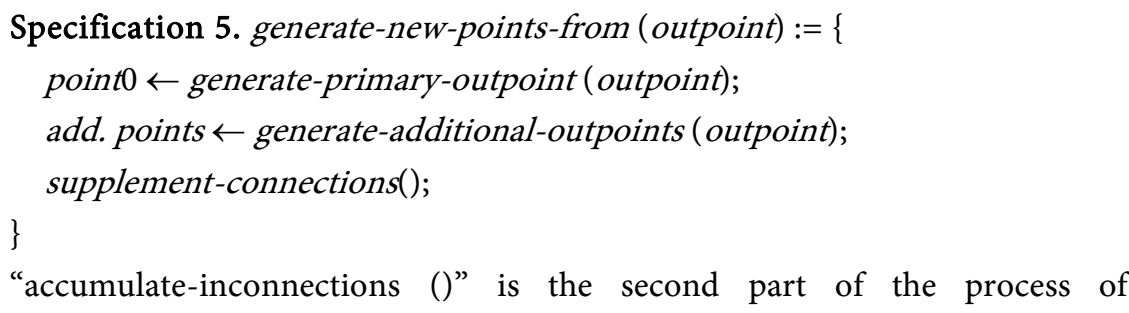
space-state-progression. The affected space points are temporarily marked as INPOINTs. The gravitation-strengths associated with the possibly multiple inward-connections are accumulated into a single value. Likewise, the emergence-direction of the space point is determined by vector summation. The emergence state of the new space point is set as the OUTPOINT.

\subsection{The Emergence of Space from a Single Source}

In the model of spacetime dynamics, it is assumed that the emergence of spacetime originates from some minimal source, which constitutes a minimal space object and continues to extend the space object via the addition of further layers of space points in each state update cycle. This means that the radius of the surface of the space object grows at each suti by $\Delta$ radius $=l_{\text {suti }}=s u t i \cdot c$. After $n$ time intervals the radius $r=n \cdot l_{\text {suti }}=n \cdot l_{\text {connection }}$.

The surface and the circumference of the space object also increase; however, the circumference of the surface is not necessarily given by circumference $=2 \pi r$, as in Euclidean space, since the length of the circumference may be dilated due to Propositions 5 and 7. According to Proposition 7, the length of the circumference is determined by the number of space points nsp(circumference)) through which a path around the circumference passes:

$$
\text { circumference }=n \operatorname{sp}(\text { circumference }) \cdot l_{\text {connection }}
$$

Proposition 8 states that "lengths within the gravitational field are dilated by the factor $F_{1}$ ". This applies also to the circumference around the space object that emerges from a single source, i.e.,

$$
\text { circumference }=2 \pi r \cdot F_{1}(r)
$$

The space emergence algorithm needs to ensure that both Equations (4) and (5) are satisfied for each new emerging surface:

$$
\begin{gathered}
n s p(\text { circumference }) \cdot l_{\text {connection }}=2 \pi r \cdot F_{1}(r) \rightarrow \\
n s p(\text { circumference })=2 \pi r \cdot F_{1}(r) / l_{\text {connection }} .
\end{gathered}
$$

The space emergence algorithm has to accomplish a growing number of surface space points $k_{p}$ according to Equation (6).

\subsubsection{The Initial Space Object}

The space objects generated by the alternative expansion algorithms depend to a minor degree on the assumed initial minimal space object. Several alternatives for the initial space object are analysed here (see Figure 2). Alternatives (a) and (b) in Figure 2, the single tetrahedron and double tetrahedron, were considered 


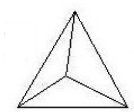

(a)

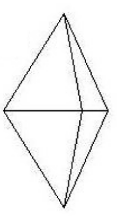

(b)

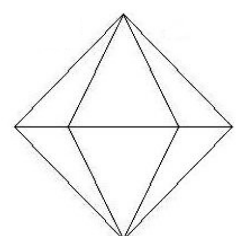

(c)

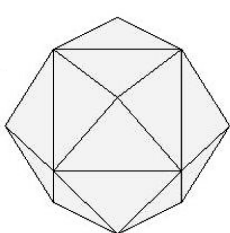

(d)

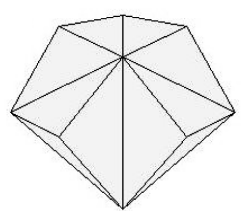

(e)

Figure 2. Alternative initial space objects.

here because they are close to CDT; however, they were rejected since they require too many expansion steps to become fairly symmetrical space objects. Alternative (d) was finally chosen as the initial space object for further analysis.

As can be seen from Figure 2, the space points at the surfaces of the initial space objects are interconnected in such a way that surface triangles can be identified. The number of surface triangles $k t$ is the major parameter that distinguishes the alternative initial space objects. In the space expansion algorithms analysed here (see below), the composition of the surface by surface triangles is maintained. Like the number of surface space points $k p_{i}$ (i.e., the number of space points at surf $\left.\mathrm{cace}_{i}\right)$, the number of surface triangles $k t_{i}$ grows with each expansion step.

$$
k p_{i+1}>k p_{i}, k t_{i+1}>k t_{i} .
$$

In the space expansion algorithms investigated here, the number of surface triangles $k t_{i}$ grows by a constant expansion factor $\eta$.

$$
k t_{i+1}=\eta k t_{i}
$$

Two examples of space expansion algorithms, with expansion factor $\eta=2$ or 3 are described in the following.

\subsubsection{Expansion Factor $\boldsymbol{\eta}=3$}

This was the first expansion algorithm investigated, since it is the simplest. At every expansion step, each surface triangle is split into three parts. Figure 3 shows that the shape of the surface triangles quickly becomes irregular, which may be a disadvantage. However, we note that we are dealing with curved space (since the expansion algorithm needs to generate curved space), which can be visualised only incompletely in Figure 3. The space curvature arises mainly from Proposition 5, which states that the length of the connections between the spacepoints, $l_{\text {connection }}$ (i.e., the sides of the triangles), is a constant of nature.

A more serious disadvantage of the expansion algorithm with $\eta=3$ is that the number of surface space points $k p_{i}$, and thus the number of space points at the circumference, nsp (circumference), grows relatively fast, and faster than Proposition 6 probably allows. This is expressed in Table 1 in the "circumference" column, and more clearly in the " $c i r_{i} / r_{i}$ " column. In Euclidean space $c i r_{i} / r_{i}$ must be $2 \pi$; however, in the space that emerges in a gravitational field, near the gravitational source $\mathrm{cir}_{i} / r_{i}$ must be greater than $2 \pi$, according to Proposition 6 . 


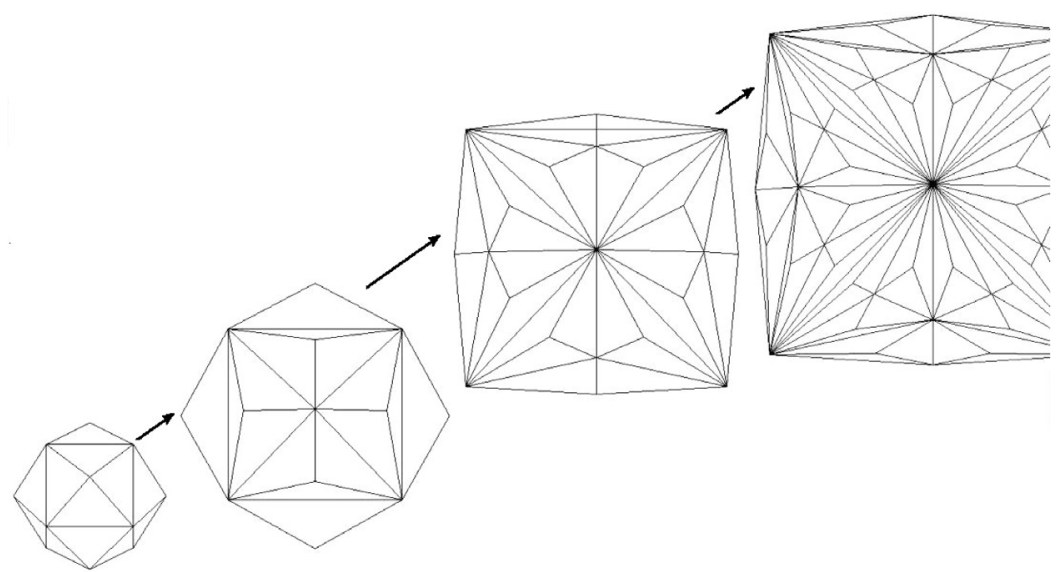

Figure 3. Emergence of space, expansion factor $=3$.

Table 1. Space expansion surfaces, expansion factor $=3$.

\begin{tabular}{ccccccc}
\hline $\begin{array}{c}\text { Layer } \\
\text { number }\end{array}$ & $\begin{array}{c}\text { surface } \\
\text { triangles, } k t\end{array}$ & $\begin{array}{c}\text { surface } \\
\text { points, } k p .\end{array}$ & $\begin{array}{c}\text { total points, } \\
k p t\end{array}$ & $\begin{array}{c}\text { radius, } \\
r_{i}\end{array}$ & circumference & $\mathrm{cir}_{i} / r_{i}$ \\
\hline 0 & 24 & 14 & 15 & 1 & 4 & 4 \\
1 & 72 & 38 & 53 & 2 & 12 & 6 \\
2 & 216 & 110 & 163 & 3 & 20 & 6 \\
3 & 648 & 326 & 489 & 4 & 28 & 7 \\
4 & 1944 & $972 ?$ & $1463 ?$ & 5 & 36 & 7 \\
$\ldots$ & $\ldots$ & $\ldots$ & $\ldots$ & $\ldots$ & $\ldots$ & $\ldots$ \\
12 & $6,377,292$ & $3,188,648$ & $12,754,596$ & 13 & $\ldots$ & $\ldots$ \\
13 & $19,131,876$ & $9,565,940$ & $38,263,764$ & 14 & $\ldots$ & $\ldots$ \\
14 & $57,395,628$ & $28,697,816$ & $114,791,268$ & 15 & $\ldots$ & $\ldots$ \\
$\ldots$ & $\ldots$ & $\ldots$ & $\ldots$ & $\ldots$ & $\ldots$ & $\ldots$ \\
i & $3 \cdot k t_{i-1}$ & $k p_{i-1}+k t_{i-1}$ & $k p_{i}+k p t_{i-1}$ & $i+1$ & & \\
\hline
\end{tabular}

\subsubsection{Expansion Factor $\eta=2$ (See Figure 4)}

In an algorithm resulting in an expansion factor $\eta=2$, the number of surface triangles $k t_{i}$, the number of surface points, $k p_{i}$, the circumference and the circumference divided by the radius grow more moderately than for $\eta=3$ (see Table 2). Depending on the mass of the minimal gravitational source, the increase in the length dilation may be too small. In any case, the growth in the number of surface triangles $k t_{i}$ and of the number of surface space points $k p_{i}$ has to be such that Equation (6) is satisfied. This cannot be achieved with a constant expansion factor $\eta$. With an increasing radius $r \rightarrow \infty, F_{1}(r)$ will approach $F_{1}(r) \rightarrow 1$, meaning that $\eta \rightarrow 1$.

The function generate-new-points-from (sp.point $[i])$ contained in Specification 4 must generate new space points such that Equation (6) is satisfied, at least for medium- and large-scale radii. Deviations from Equation (6) are unavoidable at small radii. 


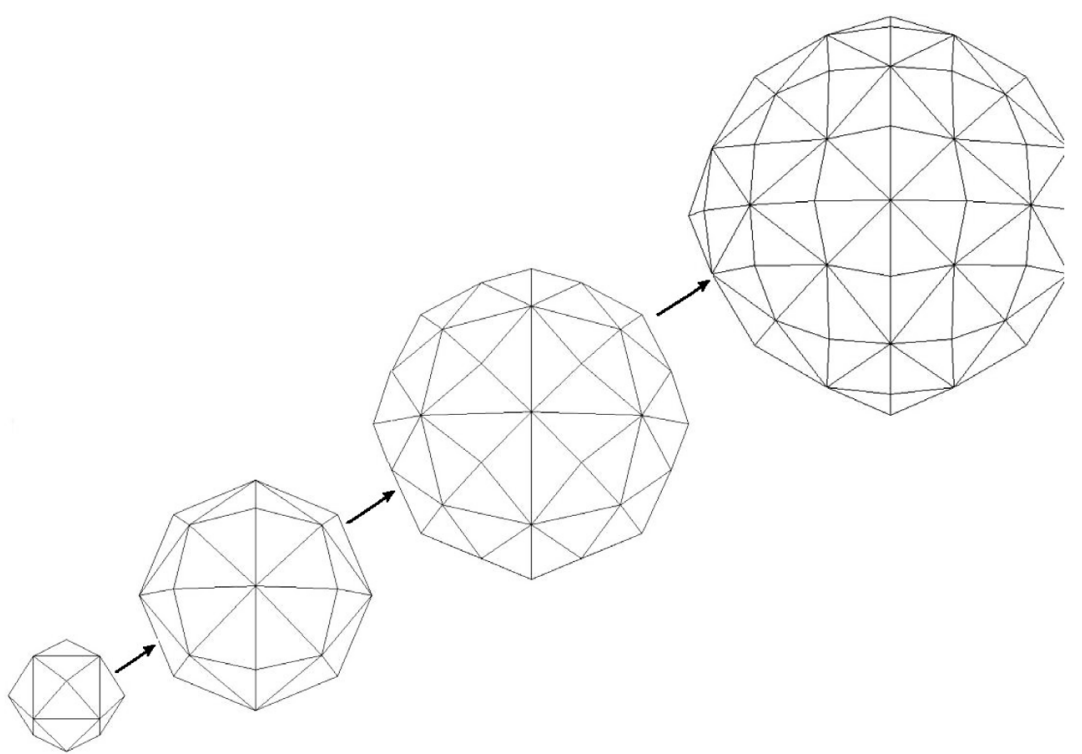

Figure 4. Emergence of space, expansion factor $=2$.

Table 2. Space expansion surfaces, expansion factor $=2$.

\begin{tabular}{ccccccc}
\hline $\begin{array}{c}\text { Layer } \\
\text { number }\end{array}$ & $\begin{array}{c}\text { Surface } \\
\text { triangles, } k t\end{array}$ & $\begin{array}{c}\text { Surface } \\
\text { points, } k p\end{array}$ & $\begin{array}{c}\text { Total } \\
\text { points, } k p t\end{array}$ & $\begin{array}{c}\text { Radius, } \\
r_{i}\end{array}$ & Circumference & $c_{i} / r_{i}$ \\
\hline 0 & 24 & 14 & 15 & 1 & 4 & 4 \\
1 & 48 & 26 & 41 & 2 & 8 & 4 \\
2 & 96 & 50 & 91 & 3 & 8,12 & 3,4 \\
3 & 192 & 98 & 189 & 4 & 16 & 4 \\
4 & 384 & 194 & 383 & 5 & 16 & 4 \\
$\ldots$ & $\ldots$ & $\ldots$ & $\ldots$ & $\ldots$ & $\ldots$ & $\ldots$ \\
12 & $6,377,292$ & $3,188,648$ & $12,754,596$ & 13 & $\ldots$ & $\ldots$ \\
13 & $19,131,876$ & $9,565,940$ & $38,263,764$ & 14 & $\ldots$ & $\ldots$ \\
14 & $57,395,628$ & $28,697,816$ & $114,791,268$ & 15 & $\ldots$ & $\ldots$ \\
$\ldots$ & $\ldots$ & $\ldots$ & $\ldots$ & $\ldots$ & $\ldots$ & $\ldots$ \\
i & $2 \cdot k t_{i-1}$ & $2 \cdot k p_{i-1}-2$ & $k p_{i}+k p t_{i-1}$ & $i+1$ & & \\
\hline
\end{tabular}

\subsection{Changes in Space Curvature Caused by a Single Source}

Space changes due to changes in the gravitational source (for example, movement of the gravitational source) arise from a very similar process to that described above in Section 4.1. The main difference from the process of emergence of spacetime is that in the space change process, a space already exists that is modified step by step.

\subsection{The Accumulation of Space Changes Caused by Multiple Sources}

For multiple gravitational sources, the space changes propagating from these 
multiple sources begin to overlap and accumulate after a certain initial phase. Three phases can be distinguished in the space emergence process:

- Phase 1: The space changes caused by the different sources propagate independently in the manner described in Sections 4.1 and 4.2.

- Phase 2: The space changes caused by the different sources begin to accumulate. The degree of overlap grows until the collection of the masses $M_{1}, M_{2}, \cdots, M_{n}$ of the multiple sources can be substituted by a singe mass $M_{\text {accum }}=\sum_{i=1}^{n} M_{i}$ located at the centre of mass.

The gradual accumulation of the gravitation caused by multiple sources is a process that is known in Newtonian mechanics.

- Phase 3: When the accumulation process is complete, the further emergence of the space (and of the gravitational field) continues via the single source process described in Sections 4.1 and 4.2.

Thus, only Phase 2, the gradual increase of the accumulation, is new compared to the processes described in Sections 4.1 and 4.2. Until Phase 2 (i.e. the accumulation phase) is complete, the major differences from the single source processes described in Sections 4.1 and 4.2 are as follows

- The space change accumulation does not start from a symmetrical minimal space object, but from a union of space objects of differing shape.

- The common space object does not represent a sphere.

- The common space object does not have a smooth surface.

- Equation (6) is not satisfied.

In summary, a number of irregularities and non-smooth developments are apparent. The major objective of the accumulation process and the algorithm is to make sure that these irregularities vanish at the end of Phase 2.

\subsection{Generate-Additional-Outpoints (p1)}

Specification 5 contains the function generate-additional-outpoints ( $\mathrm{p} 1)$. This is a key function with the emergence of spacetime. The algorithm has to satisfy the following objectives:

- GRT compatibility at medium and large scale.

Maximum GRT compatibility is the general goal for the construction of the causal model of spacetime dynamics. The algorithm for the function generate-additional-outpoints ( $\mathrm{p} 1$ ) has the major responsibility for ensuring that the number of additional space points that are generated during the space change propagation process satisfies Equation (6)

$$
n s p(\text { circumference })=2 \pi r \cdot F_{1}(r) / l_{\text {connection }}
$$

Because of the discreteness of the number of space points that consitute the surface of the expanding space points, deviations from Equation (6) are unavoidable. Additionally, more significant deviations, occur at the very small scale; that is they occur near to the minimal sources, the quantum objects.

- Background independence - space point locality. 
Background independence is typical aimed for with theories and models of spacetime. It means that the theory/model should not depend on any preexisting spacetime structure and contents such as coordinates or curvature. In the context of a local causal model, the requirement is strengthend to also request space point locality. Space point locality means that the algorithm/function which determines the progression of the system state (i.e., generate-additional-outpoints()) must not depend on any non-local parameters. The dependency of Equation (6) on the radius $r$ in $F_{1}(r)$ disturbs the space point locality. Thus, the dependency on $r$ must be transformed into a dependency on some local space point component such as, for example, the gravitation-strength (see Definition 3).

Generate-additional-outpoints ( $\mathrm{p} 1$ ) generates $n_{\text {add }}$ additional new space points that are connected to space point $p 1 . n_{\text {add }}$ is an integer number, $n_{\text {add }} \geq 0 \quad\left(n_{\text {add }}=0\right.$ means that the number of space points that emerge from $\mathrm{p} 1$ does not grow). In general, a "new" spacepoint may be shared by multiple source space points $p 1_{1}, p 1_{2}, \cdots$. This means that the new space point is connected to multiple source space points $p 1_{1}, p 1_{2}, \cdots$.

\section{Applications of the Model of Spacetime Dynamics to Quantum Field Theory}

In Sections 3 and 4, a causal model of the dynamics of spacetime has been described. According to the model, spacetime changes (i.e. the gravitational field) continuously propagate from the minimal sources, called quantum objects. In quantum field theory (QFT), the quantum objects are also the sources of additional dynamical processes. Quantum objects are the sources of virtual particle fluctuations. The movement of quantum objects through space, is described in terms of paths that constitute the wave function. Also, particle scattering in QFT is described in terms of paths for virtual particles that lead to a range of probability amplitudes for different possible scattering results. Considering the various cases of the dynamics in QFT, the question arises on how QFT (virtual particle) paths relate to the model of spacetime dynamics described in the preceding sections. As with the general subject, the question can be asked in two parts:

1) How do the dynamics of quantum fields (including quantum objects) relate to the elementary structure of spacetime described in Section 3 ?

2) How do the dynamics of quantum fields (including quantum objects) relate to the model of the dynamics of spacetime described in Section 4 ?

Question 1 requests a detailed answer in order to demonstrate that the model of spacetime dynamics can also be applied to the dynamics of quantum fields and quantum objects. The details are straight forward, yet non-trivial. The answer to question 2 is less direct. Integrating quantum field dynamics and spacetime dynamics at different degrees are imaginable, ranging from minimal integration (i.e., adaptation to the proposed spacetime structure only) to maximal integration (i.e. a combined model for both subjects as for example quantum gravi- 
ty aims for). In Section 5.2 the model proposed by the author is described.

\subsection{Mapping Quantum Fields and Quantum Objects to the Elementary Structure of Spacetime}

The task is to map the parameters and components that constitute a quantum field or a quantum object to the parameters and components of the spacetime as described in Section 3. In Section 3.1, the integrated view of spacetime (as assumed in standard GRT) is described as being disturbed by the strict separation of space and time implied by the causal model. This may be considered as too restrictive for a GRT-compatible model of spacetime to be applied to QFT. However, starting with a topology where space and time are separated into $\Sigma \times R$ where $\Sigma$ is a three-dimensional manifold and $R$ is a line, is a popular approach with theories directed toward quantum gravity (see [7] on loop quantum gravity). It leads to the so-called "Hamiltonian formulation of general relativity" (see [7]). As with the model of spacetime dynamics described in this article, the integrated view of spacetime is restored by processes that relate the spatial changes to the progression of time (e.g., by a causal model).

In Section 3 Definition 2, space is defined as consisting of interconnected space points and a space point is defined as

spacepoint $:=\{\psi$, gravitation spec; connections $\}$;

Here, fields are represented by the component $\psi$. (Whether $\psi$ refers to a single type of field or to possibly multiple field types is here left open.) In [8], quantum objects are defined as composite objects consisting of 1 to $\mathrm{n}$ particles.

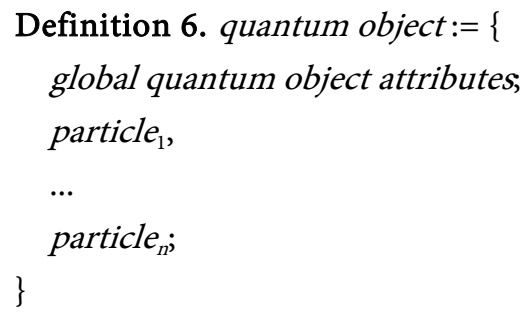

The particle encompasses a set of spacepoints and global particle attributes:

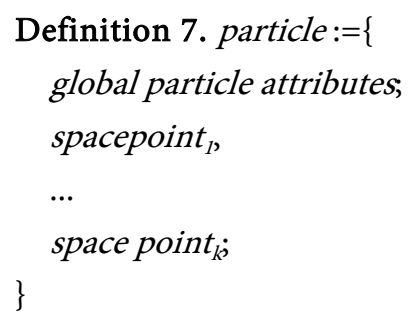

Examples of global attributes (global quantum object attributes and global particle attributes ) are mass, charge, spin, etc. With the specification of a local causal model at a specific level of detail, the inclusion of the global attributes may disturb the provision of a local causal model. Therefore, in a detailed local causal model, the global attributes may have to be supported by aggregation processes and/or collective behaviour processes (see Section 5.3 Collective behaviour). 


\subsection{Mapping of the Dynamics of Quantum Fields and Quantum Objects to the Dynamics of Spacetime}

The model that is roughly described as follows is based on two types of work:

1) Loop quantum gravity [7] and its descendants comprising spin networks (see [10]), spin foam (see [11]), and causal dynamical triangulation [1].

The coupling of the dynamics of space (e.g., the propagation of space changes) with the dynamics of quantum fields and particles is an idea that has already been pursued with causal fermion systems (see [12]).

2) In [13] and [8] a causal model of QT/QFT is proposed where the physics of QT/QFT is confined in "quantum objects". The refinement and an improved foundation of the model described in [13] and [8] was determined to require a causal model of spacetime dynamics. The causal model of spacetime dynamics described in Sections 3 and 4 has been developed as an attempt to fulfill this requirement.

\subsubsection{The Movement of Objects within Space}

According to GRT, the movement of objects within space follows the geodesics of the space. This means, two parameters determine the path of the object: 1) the objects momentum and 2) the structure of the space, in particular, the curvature of the space.

With the model of spacetime dynamics, especially when applied to quantum theory, the GRT-based model of object movement has to be adjusted and refined for two aspects: 1) the term geodesics must be redefined for discrete granular paths, and 2) the momentum of quantum objects in general does not have a single definite value, but a range of (possible) values. Regarding these two aspects resulted in the following model for the movement of objects within space.

- The moving object is represented by the space content $\psi$ of a set of space points (see Definition 2).

- Part of $\psi$ is the momentum vector component $p$.

- When the propagation process reaches a space point $\mathrm{sp}$, the momentum vectors from the in-connections of sp are summarized to a single consolidated momentum vector.

- The consolidated momentum vector is then distributed to the out-connections.

- The distribution is such that the out-connection(s), which matches best the direction of the consolidated momentum vector, obtains the largest part of the consolidated vector.

Given the aforementioned schema, the following types of object movements may be distinguished:

1) Classical straight forward movement following a single definite geodesic,

2) Quantum movement with a network of paths and with different probability amplitudes,

3) Loops 
a) Classical loops according to a geodesic that represents a loop (examples: planets and satellites),

b) Quantum loops: Loops resulting from quantum objects and constituting quantum objects.

Quantum movements and quantum loops are further described in the following.

\subsubsection{Quantum Movement}

In Section 4, the dynamics of spacetime is described as involving the summation of the in-connections of a space point followed by the distribution of the aggregated effect to the multiple out-connections. A similar operation is also known with the operator equations of QFT (see, for example [14]). Two virtual particles may join and annihilate each other to create a single new virtual particle of a specific type; or vice versa, a single virtual particle may be annihilated resulting in the creation of two new virtual particles of specific types. The graphical representation of the possible annihilate/create (or join/split) operations is given by Feynman diagrams. In quantum electrodynamics (QED), the operator equation for the creation and annihilation of the field has the form (see [14]):

$$
H_{W}(x)=-e N\left\{\left(\bar{\psi}^{+}+\bar{\psi}^{-}\right)\left(A^{+}+A^{-}\right)\left(\psi^{+}-\psi^{-}\right)\right\}_{x}
$$

where $\psi^{+}, \psi^{-}, \bar{\psi}^{+}, \bar{\psi}^{-}, A^{+}, A^{-}$are the creation and annihilation operators for electron, positron and photon. This leads to the eight fundamental Feynman diagrams shown in Figure 5. The operator combination of QFT (normally) applies to three operations (two creates and one annihilate or one create and two annihilate). For a mapping of the QFT processes to the model of spacetime dynamics, the QFT operations have to be mapped to the $n$ in/out connections of the space point. A typical space point has $\mathrm{n}=14$ connections. This enables the use of various strategies (i.e. algorithms) for the mapping of the three lines of a fundamental Feynman diagram to the 14 space point connections. For the application of the model of spacetime dynamics to quantum fields, the overall strategy is the preservation of the number of fermion in-connections and fermion-out connections and the allowance of additional boson connections. This enables the types of QED space point connections shown in Figure 6. (For practical purposes only part of the boson connections are shown in Figure 6). The cases that correspond to the QED first order diagrams shown in Figure 5 are the cases (1) to (3) in Figure 6. Case (4) and case (5) support an increased diversity of the possible fermion and boson paths.

Notice that the mapping of the QFT operations to the in/out connections of the space points is part of the dynamical QFT processes (it is not a static mapping).

The utilization of the complete set of in/out connections for the join/split operation on (virtual) particle paths delivers the equivalent to the superposition of paths which in QFT is expressed by the path integral. In standard QFT (see [15]), the path integral is written as $K(b, a)=\int_{a}^{b} \mathrm{e}^{(i / \hbar) S[b, a]} D x(t)$. 

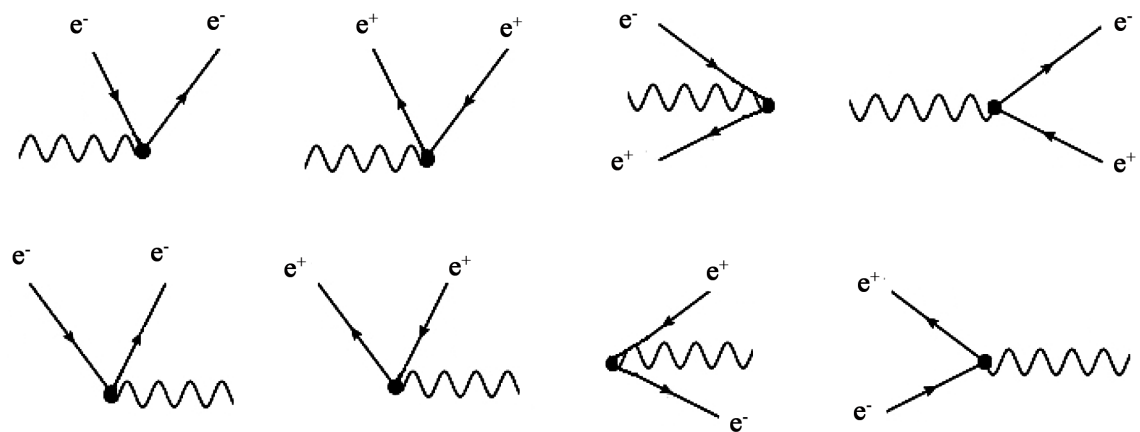

Figure 5. Fundamental Feynman diagrams of quantum electrodynamics.

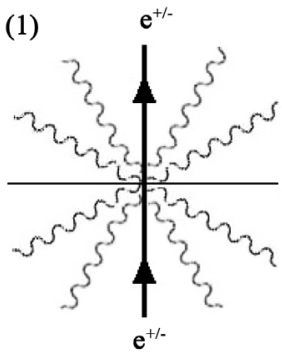

(2)

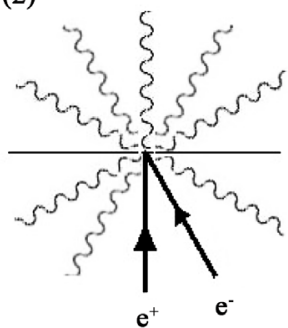

(5)
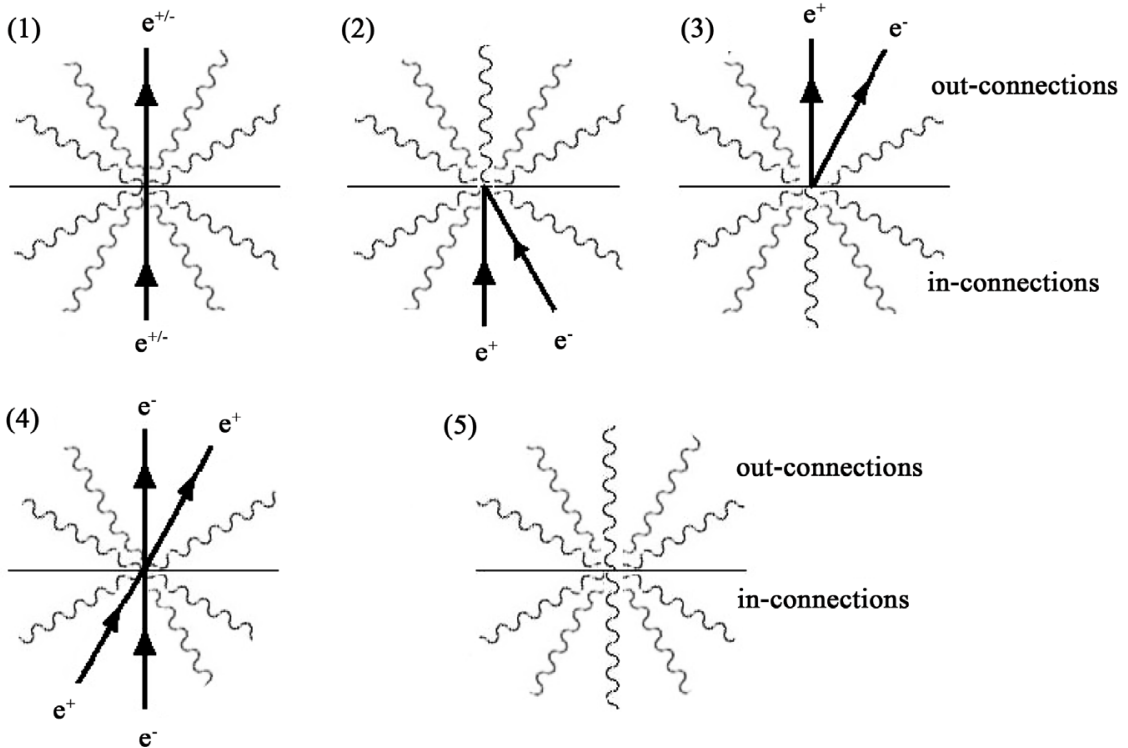

Figure 6. Possible QED connections of a space point.

The discreteness of the model parameters (space, time and paths) may results in significant incompatibilities at the very small scale. The discreteness of the model parameters in conjunction with the local causal model eliminates the need for renormalization (if a suitable algorithm for the assignment of in/out connections is applied).

\subsubsection{Quantum Loops}

In terms of a causal model, a physical object moves into a loop, if two conditions are satisfied:

1) The object moves in a spatial environment that enables geodesic loops.

2) The object has reached a recurrence state, i.e., a state such that the causal progression of the object may lead to a recurrence of this object state.

Geodesic loops can occur only if space has a specific curvature. The simplest example of space curvature that enables geodesic loops are the spherical surfaces that develop with the emergence of space caused by one or multiple sources (see Sections 3 and 4). With the model of spacetime dynamics, the emergence of spatial changes occurs through the successive addition of spherical surfaces. The 
spherical surfaces occur already around the minimal sources, i.e., the quantum objects. As described above (see Quantum movement), in contrast to GRT, where the geodesics are single lines, in the model proposed, the geodesic consists of a network of paths with splits and joins at each space point according to the rules and diagrams of QFT. This holds true also for the geodesic loops. Because of the large number $\mathrm{n}$ of in/out connection $(n>3, n \sim 14)$, there may be paths of the network that do not end up in the loop. In general, there will be open ends (see Figure 7).

In the quantum loop shown in Figure 7, the in/out connections are labeled by specific symbols. In Figure 7 the labels (and thus the paths) refer to (virtual) particle types of QED $\left(\gamma, \mathrm{e}^{-}, \mathrm{e}^{+}\right)$. This emphasizes the close relationship between the quantum loop network and Feynman diagrams. An alternative labeling of the paths, and thus an alternative interpretation of the quantum loop, would be to show the similarity with spin networks. With spin networks, the connections (i.e., line segments) within the network are attributed by spin numbers (with the original introduction by R.Penrose [10]) or the dimension of the parallel transport matrix (see [7]). Similar to spin networks, the quantum loop network defines the possible paths of state transitions including possible final result state (i.e., the recurrence state). If an extra (logical) dimension is added to the quantum loop network (or to the spin network) to show the complete multitude of possible networks that support a specific recurrence state, the equivalent to the spin foam (see [11]) is given.

\subsection{Collective Behaviour}

One of the objectives of the causal model presented in this article is that the model should be a local causal model. The target space-point-locality is damaged by the inclusion of composite quantum objects with object-global attributes (e.g.

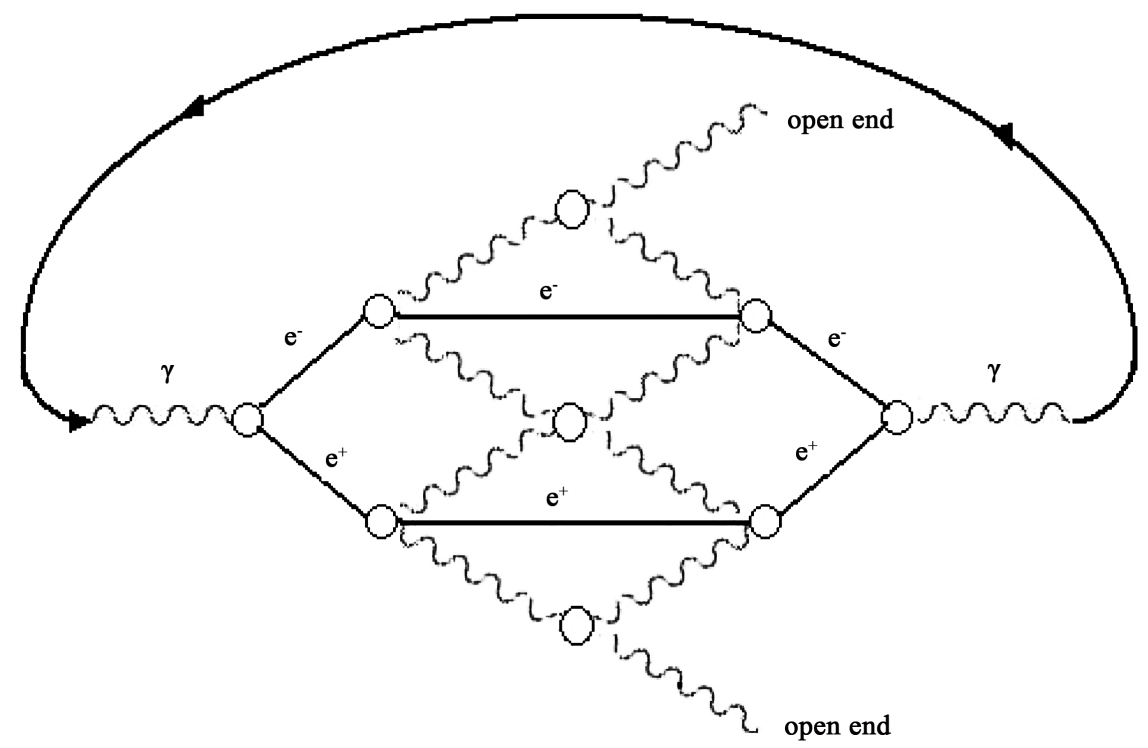

Figure 7. A quantum loop containing a network of paths. 
mass and spin) and instantaneous processes (e.g., the collapse of the wave function and entanglement), if it is not possible to break down the formation of the composite objects and the related non-local effects to space-point-local state transitions. In the causal model of QT/QFT described in [16], the non-local effects are explained by the collective behaviour of spacetime elements. Based on the causal model of spacetime dynamics described in Sections 3 and 4 and the concept of the quantum loops, the model described in [16] can now be refined as follows.

The formation of (semi-) stable quantum objects (elementary as well as composite quantum objects) is a collective behaviour process in form of a quantum loop that runs within the (small) area of curved space around the components of the quantum object.

As the described collective behaviour process represents a model for the emergence of quantum objects and the related quantum-object-global attributes, the disturbance of this collective behaviour process provides a possible model for the instantaneous non-local QT/QFT processes such as particle decay, the collapse of the wave function, and decoherence. The model which describes the emergence of a quantum object as a collective behaviour process has many similarities with G. Groessing's proposal to explain the emergence of a quantum system as a self-organization process (see [17]).

\section{Applications of the Model of Spacetime Dynamics to Cosmological Dynamics}

In addition to enabling alternative interpretations and models of QFT in curved spacetime (the topic of Section 5), the proposed model of spacetime dynamics also leads to (possible) new interpretations and models of cosmological dynamics. The main features of the model of spacetime dynamics that enable/demand new interpretations are: 1) gravitational length dilations and 2) the non-smooth aggregation of spacetime dynamics.

\subsection{Gravitational Length Dilations}

In Section 3.1, Proposition 9 states that in the proposed model of spacetime dynamics, clock rate dilation is considered a secondary effect caused by the primary effect, the length dilation due to space curvature, i.e., due to the gravitational field. According to Section 3.1, the lengths (and, as a secondary effect, also the clock rates) around a gravitational source are dilated by the factor $F_{1}=\sqrt{1-\frac{2 G M}{c^{2} r}}$.

This means, that if at a spacepoint near the gravitational source, at radius $r 1$, the dilation factor is $F_{1}=\sqrt{1-\frac{2 G M}{c^{2} r 1}}$, at a spacepoint that is farther away from the source, at radius $r 2(r 2>r 1)$, the dilation factor gets closer to its maximal value, 1 . Since the radius $r$ is only dilated by a factor $F_{r}<F_{1}$, this means that, 
contrary to Euclidean geometry, the circumference $C$ of an orbit around a gravitational source is greater than $2 \pi r$.

The dilation of the circumference is an effect which cannot be directly observed by an observer such as an astronomer. In the projection of the orbit to a picture in Euclidean geometry, the relation between the observed radius $r_{o}$ and the observed circumference $C_{o}$ is still $C_{o}=2 \pi r_{o}$. The orbital length dilation can be observed only indirectly, by examining the dynamics of objects orbiting around a gravitational source. For example, the velocity of objects orbiting a gravitational source will show deviations from the laws of Newtonian dynamics. The most famous examples of unexpected deviations from Newtonian dynamics in cosmological observations are the "flat galaxy rotational curves", for which gravitational length dilations offer a possible explanation (see below).

If, for two neighboring spacepoints at equal radius $r=r 1$, the non-dilated distance between them is $d_{N}$, the dilated distance $d_{d}$ is $d_{d}=d_{N} \cdot F_{1}(r 1)$.

(The subscript $\mathrm{N}$ stands for "Newtonian" or "non-dilated", d stands for "dilated", and o stands for "observed")

For larger distances, the overall dilated path length following the spacepoints $s p_{1}, \cdots, s p_{k}$ is

$$
p l_{d}\left(s p_{1}, \cdots, s p_{k}\right)=\sum_{i=1}^{k-1} l \cdot \operatorname{connection}\left(s p_{i}, s p_{i+1}\right) \cdot F_{1}(r 1)
$$

For paths on a (Schwarzschild metric) circumsphere, i.e., with constant radius $r$, this can be simplified to

circumspherepl $_{d}\left(s p_{1}, \cdots, s p_{k}\right)=F_{1}(r) \cdot$ path $_{N}\left(s p_{1}, \cdots, s p_{k}\right)$ by setting $\operatorname{path}_{N}\left(s p_{1}, \cdots, s p_{k}\right)=\sum_{i=1}^{k-1} l \cdot \operatorname{connection}\left(s p_{i}, s p_{i+1}\right)$.

In Equation (7), the dilated path length is obtained by multiplying the "non-dilated" path length by the factor $F_{1}$, leaving aside that always $F_{1} \leq 1$. In order to avoid misinterpretations and use a more meaningful base for the dilation factor, the dilation factor $F_{2}$ is introduced such that

$$
\text { circumspherepl }_{d}\left(s p_{1}, \cdots, s p_{k}\right)=F_{2} \cdot \text { path }_{N}\left(s p_{1}, \cdots, s p_{k}\right)
$$

and $F_{2}$ is defined as $F_{2}(r)=F_{1}(r) / F_{1}(r 0)$, with $r_{0}$ being the minimal radius (e.g., $\left.r_{0}=1\right)$. This ensures that $F_{2}$ is always $F_{2} \geq 1$.

In radial direction, matters are more complicated because the factor $F_{2}$ varies with increasing radius. Let us define that instead of the factor $F_{2}$, the dilated length in radial direction is dependent on a factor $F_{3}$

$$
\text { radialpl }_{d}\left(s p_{1}, \cdots, s p_{k}\right)=F_{3} \cdot \operatorname{path}_{N}\left(s p_{1}, \cdots, s p_{k}\right) .
$$

The difference between $F_{3}$ and $F_{2}$ depends on several parameters. (For the special case of galactic rotational curves, the parameters are described in Section 6.3.) In general, $F_{3}<F_{2}$. This means that for a sphere with radius $r_{d}$ around a gravitational source the dilated circumference $c_{d} \neq 2 \pi r_{d}$.

The Observation of Space Distortion by a Distant Observer

For the analysis of the implications of the gravitational length dilations for cos- 
mological models, it is important to analyze the extent to which the length dilations can be observed by a distant observer, such as an astronomer. The (3-dimensional) space distortion due to non-uniform length scale (like other space curvature) can hardly be directly observed. It can be indirectly observed, by observing an objects movement within a strong gravitational field or by observing the large-scale results of dynamical space(-time) processes. Astronomers who observe the cosmos typically obtain projections of 3-dimensional curved space configurations to 2-dimensional images. Assuming that the $\mathrm{Z}$-axis points to the observer, the projections apply to the $(\mathrm{X}, \mathrm{Y})$-plane. The length dilations in radial directions (from the gravitational source) also appear in the projections; that is, they can be observed. As described above, lengths in radial directions are dilated by the factor $F_{3}$.

The dilation of lengths that are not purely in radial direction will also appear in the observations, but only to the extent of the radial direction dilation. For example, according to the description given above, the orbit around a gravitational source is dilated by the factor $F_{2}\left(F_{3} \leq F_{2}\right)$. The distant observer, however, will see the length of the orbit as $r_{d} \cdot 2 \pi$, with the dilated radius $r_{d}=$ non-dilated-radius $F_{3}$.

Furthermore, the length projections for an area of the cosmos (i.e., observations) must be seen in relation to the space distortions in the surrounding space.

\subsection{Non-Smooth Aggregation of Spacetime Dynamics}

In cosmology, it is well known that the strength of the gravitational field within a (dense) gravitational object increases in a different manner with increasing distance from the centre of mass than is the case outside the object. Section 4.3 explains that the process of aggregation of spacetime curvature changes resulting from several sources may be even more complex than assumed in the standard models. This may affect various aspects of the cosmological models.

The features of the proposed model of spacetime dynamics described above may have implications for many aspects of the present standard cosmological model. On the positive side, these features also offer opportunities for new explanations and interpretations in areas of cosmology that are not yet sufficiently understood. The major areas identified by the author in which the application of the model of spacetime dynamics may result in new explanations of cosmological observations are the following:

\subsection{Flat Galaxy Rotational Curves and Dark Matter}

The existence of "dark matter" has been proposed as a possible explanation of the observed flat galaxy rotational curves, while an alternative explanation known as modified Newtonian dynamics (MOND) has also been proposed. Two further proposed theories explain the flat rotational curves by the existence of a new force: 1) a so-called entropic force (see [18]) or 2) a so-called gravo-inductive field (see [19]). 
Gravitational length dilation (see above) may provide yet another possible explanation for the flat galaxy rotational curves, in which the length and clock rate dilation (i.e. time dilation) yield a velocity larger than that deduced from the observed rotational curves. In simpler terms, the flat rotational curves are observed due to the spacetime curvature, in which the orbit has a larger length dilation than the radius.

Let us consider a galaxy with rotational curves that are in accordance with (unmodified) Newtonian dynamics. The velocities of circular orbits are determined by Newtons force law $M a=F$ for a test particle with mass $M$ and acceleration a. For the rotational curves of stars of a galaxy, two phases are distinguished with respect to the velocity $\mathrm{v}$ of circular orbits (see Figure 8$)^{3}$. In phase-1, when the star is within (or close to) the "bulge" that surrounds the center of the galaxy, the velocity according to Newtonian dynamics is

$$
v(r)=\sqrt{\frac{G M(r)}{r}} .
$$

$G$ is the gravitation constant, $\mathrm{r}$ the radius, and $M(r)$ the mass, which is dependent on the radius. Resolving the dependency of the mass on the radius by application of the density law $M(r)=\rho_{0} V$ (see [20]), results in $v(r)$ being proportianal to $\mathrm{r}$ :

$$
v(r) \sim r .
$$

When the distance from the bulge is sufficiently large phase- 2 applies where the velocity is expected to be

$$
v(r) \sim \sqrt{\frac{1}{r}} .
$$

For phase-1, the observations are in agreement with the expectation. Phase-2 presents a problem. Instead of the decreasing velocity (Figure $8, v_{N}$ ), according to Equation (12), a flat rotational curve is observed (Figure 8, $v_{o}$ ), i.e., the velocity observed is greater than expected.

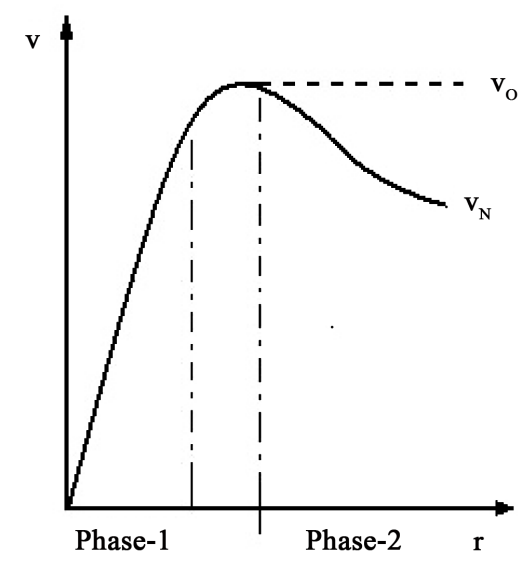

Figure 8. Velocities in galaxy rotational curves.

${ }^{3}$ Figure 8 is only a schematic figure. No attempt has been made to show correct proportions. 
In addition to the dark matter theory and the MOND theory, the length dilation assumed with the proposed causal model of spacetime dynamics may provide another explanation for the flat galaxy rotational curves. The explanation concerns, first of all, the differences in the length dilation of the circumference of the rotational curve and the length dilation of the radius. This difference is the difference between the factors $F_{3}$ and $F_{2}$, described in Section 6.1. The difference between $F_{3}$ and $F_{2}$ causes differences between the observed values and the real values of the physical parameters as described in Section 6.1.

In the context of galactic rotational curves, for the calculation of the value of $F_{3}$, the following points have to be taken into account:

1) In contrast to $F_{2}$ in Equation (8), $F_{3}$ in Equation (9) is the mean value for a path with varying radius $r$.

2) The complete range of radius to be considered includes the phase- 1 part, the phase- 2 part, and the part between phase- 1 and phase- 2 . In addition to the uncertainty as to where exactly phase- 1 ends and where exactly phase- 2 starts, the following points are difficult to quantify:

3) During phase-1, not only does $F_{2}$ (the basis for determining $F_{3}$ ) vary with $r$, but as indicated in Equation (12) the (effective) mass $M(r)$ also increases with increasing radius.

4) As described in Section 4.3, the author questions the general applicability of the density law $M(r)=\rho_{0} V$ for the determination of $\mathrm{M}(\mathrm{r})$ for the complete phase-1.

Because of these points, the author is at present not able to provide a somewhat reliable calculation of $F_{3}$ for the observed flat galaxy rotational curves. At least it is possible to state a rough relation between $F_{3}$ and $F_{2}$ :

$$
1 \leq F_{3}(r 1) \leq F_{2}(r 1) \text {. }
$$

In other words, $F_{3}$ for the path between radius $r=r_{0}$ and radius $r=r_{1}$ is greater than 1 and less than $F_{2}$ for $r_{1}$. This implies that for a circumference $c_{d}$ with radius $r_{d}, c_{d}>2 \pi r_{d}$ something that is possible only in curved space, and something that can never directly be observed by a distant observer.

The relationship between non-dilated entities, the dilated entities, and the observed entities with galaxy rotational curves is summarized in Table 3 with the three rows "w/o dilation", "dilated" and "observed". The essential table entry is the observed velocity, which is stated to be higher than the (real) dilated velocity (which is equal to the non-dilated velocity). The observed velocity $V_{o}$ is $v_{o}=c_{o} / t_{o} \cdot c_{o}$ is measured or estimated by the observer in terms of the length (scale) of the observed radius $r_{o}, c_{o}=2 \pi r_{o}$. The observed radius $r_{o}$, however, is roughly equal to the dilated radius, according to Section 6.3. Because, for the observer, the circulation time $t_{o}$ is equal to the non-dilated time $t_{N}$, the velocity $V_{o}$ appears to be greater than the expected velocity $V_{N}$. In summary, the velocity $V_{o}$ appears to be greater than the expected velocity $V_{N}$, because the length dilation effects are only partly visible to the distant observer. 
Table 3. Galaxy rotational curves.

\begin{tabular}{cccccc}
\hline Cases & $\begin{array}{c}\text { Dilation } \\
\text { factors }\end{array}$ & $\begin{array}{c}\text { Circulation } \\
\text { time, } t\end{array}$ & Circumference $c$ & Radius $r$ & Velocity $v$ \\
\hline w/o dilation & 1 & $t_{N}$ & $c_{N}=2 \pi r_{N}$ & $r_{N}$ & $v_{N}=c_{N} / t_{N}$ \\
dilated & $F_{2}, F_{3}$ & $t_{d}=t_{N} F_{2}$ & $c_{d}=c_{N} F_{2}$ & $r_{d}=r_{N} F_{3}$ & $v_{d}=v_{N}$ \\
observed & - & $t_{o}=t_{N}$ & $c_{o}=2 \pi r_{o}$ & $r_{o}=r_{d}=r_{N} F_{3}$ & $v_{o}=c_{o} / t_{o}$ \\
Example: & 1 & 100 & 628 & 100 & 6.28 \\
w/o dilation & $1.1,1.05$ & $t_{o}=t_{N}=100$ & $c_{o}=c_{N}=660$ & 105 & 6.6 \\
dilated & - & & & 105 & 6.28 \\
observed & & & & & \\
\hline
\end{tabular}

\subsection{Pioneer Anomaly}

A significant number of proposals have been published in an attempt to explain the Pioneer anomaly. [21] presents an excellent overview of the detailed nature of this anomaly and the efforts made to explain and study it. Among the possible explanations are the MOND-based explanation described above, "dark matter", and "gravitational forces due to unknown mass distributions and the Kuiper belt". Since 2012, the thermal recoil force has appeared to be the most widely accepted explanation (see [22]) for the Pioneer anomaly. As for the flat galaxy rotational curves (Section 6.3), the gravitational length dilation of the proposed model of spacetime dynamics provides a further possible explanation.

\subsection{The Expansion Speed of the Universe and Dark Energy}

According to the standard model of cosmology, the universe is continuously expanding. The method used to determine the speed of this expansion is typically a measurement of the redshift of light emitted by the most distant stars. Based on these redshift measurements, astronomers have observed an increasing speed of expansion of the universe. The explanation currently favoured by astrophysicists for this increasing speed of expansion is a combination of several causes, with the largest contribution coming from "dark energy". All of the features of the model described above that can have an impact on cosmological models (i.e. 1) gravitational length dilation; 2) the non-smooth aggregation of spacetime dynamics; and 3) collective behaviour) may contribute to the varying (i.e. increasing) speed of expansion of our universe. Further work is required to obtain rough estimates of the possible contributions of these individual features and the combination of their effects.

\section{Discussion}

\subsection{The Special Role of Time}

SRT and GRT have taught that space and time are integrated into spacetime. The major reason for taking this view is that in the laws and equations of SRT 
and GRT, time and space occur in combination, and the causal progression of the system state depends on the progression of the combination of both space and time. The causal model of spacetime dynamics presented in this article also implies a tight relationship of space and time, although with a different interpretation (see Section 3.1).

Nevertheless, there are also (good) reasons for not neglecting some fundamental differences between space and time. The major points where the concept of time assumed for the model described deviates from the time concept described (or implied) in some physics literature are:

- Arrow of time

The formal definition of a causal model (in general, not just for the model described in this article) assumes a constant direction in which time progresses, i.e., an arrow of time. Reverse progression of time or variable direction of time progression is just not supported by the model. The author believes that a causal model in general implies an arrow of time. In other words, a model that does not adhere to a unique constant direction of time would show more flexibility than nature shows in reality. The model would not be reality conformal.

- Time slices

With the goal of showing as much commonality as possible between space and time, some physics literature do not describe the extension of the time coordinate as differing from the extension of the space. In the formal definition of a causal model, the laws of physics that specify the state transitions can always access only the system state of the current point in time. It is not possible to access past or future time slices of system states. Models that would allow reference or even modifications of past or future system states are considered as (probably) not reality conformal and would be very complicated.

\subsection{Time Dilation and/or Length Dilation?}

Both SRT and GRT predict, under specific circumstances, time dilation and/or length contraction. In textbooks covering SRT and GRT, it is not always clear whether: 1) the two effects occur simultaneously, 2) the two effects are just two possible views from a non-local observer, or 3) there are cases where time dilation occurs (but no length contraction) and vice versa. For the proposed model of spacetime dynamics, length dilation is the primary effect. In the model, time dilation-more precisely, the clock rate dilation-is seen as a consequence of the length dilation. Length is a spatial attribute, while clock rate is a property of processes running in a causal subsystem. (In areas of space where there is no causal subsystem, there is no clock rate dilation, nor time dilation.) Despite the basic differences in the roles that time dilation and length dilation play (in the model), these functions are highly interrelated (see Section 3.1).

\subsection{Clock Rate Dilation as a Consequence of Length Dilation?}

Assertion 1 in Section 3.1 suggests a tight fundamental relationship between space and time. As an implication of the proposed spacetime relationship, Prop- 
osition 9 states that all gravitational time dilations predicted in GRT are a consequence of related length dilations. Gravitational length dilation appears to be a controversial subject among physicists (see [23] and various discussions in internet forums). Most physicists who interpret GRT to imply length dilation argue that the length dilation is a side effect of the time dilation, and possibly simply a local measurement effect. Time dilation without length dilation, they argue, would imply the violation of the constant speed of light for the movement of light within a gravitational potential with time dilation. The present author agrees with this argument, but favours a model in which the length dilation is the primary effect and time dilation is a secondary effect that applies purely to physical processes that are executed in a length-dilated environment. The advantages of the proposed model are as follows:

1) It enables a simpler formulation of causal models (the authors goal of developing a causal model led to the proposed reinterpretation of the spacetime relationship.)

2) It supports the notion of global simultaneity and global time, such as the age of the universe, which is useful for astrophysical theories.

3) It offers a first step towards removing the incompatibilities between GRT and QT concerning the "problem of time" (see [7]).

A possible foundation of the proposed spacetime relationship may be as follows:

Conjecture 1. All physical processes can ultimately be broken down to length-related state changes, and changes in the length scaling therefore directly result in clock rate dilations of the affected process.

\section{Conclusions}

The model of spacetime dynamics described in this article does not aim at providing another theory of the subject. Rather, it has the goal of providing a special model, namely a causal model, of the subject for which a generally agreed upon theory exists. However, it is not possible to derive a causal model of spacetime dynamics purely from GRT. GRT establishes a powerful base for the development of the model, but supplementary statements and interpretations are required to construct a somewhat complete (local) causal model of this area of physics. The described causal model is not claimed to be the only possible or valid model of the subject. Alternative models, possibly focusing on specific aspects, are imaginable. With those features of the model that could not be directly derived from GRT and where, therefore, new solutions had to be invented, it may turn out that the solutions of the present model have to be replaced by solutions that are in accordance with new experiments.

The major items, where the proposed model deviates from the standard interpretations of GRT, QFT and cosmology are:

1) The assumption of the length dilation as the primary effect of space curvature that causes clock rate dilation as a secondary effect. 
2) The assignment of additional bosonic create operators for the out-connections of space points leading to the possibility of quantum loops.

3) Gravitational length dilation as the possible explanation of the flat galaxy rotational curves.

Disregarding the uncertainties about the ultimate validity of certain details of the proposed model, there are nevertheless a number of findings that the author believes are worth noticing:

- For an area of physics, it is mandatory that the construction of models of the complete dynamics is feasible. The type of model that is best suited to describe the complete dynamics is the causal model. The lack of feasibility of constructing a causal model of a theory of physics may be considered as an indication of the incompleteness of the theory.

- As SRT and GRT show, space and time have to be viewed as integrated. The progression of time can be described only in connection with spatial state changes. The length scaling within space (including curvature) can only be described with reference to processes executing for a specific time interval. However, besides this fundamental tight relation between space and time, it is also necessary to point out the fundamental differences in the roles, structure, and properties of space and time.

Further work is required to refine the model and make the ideas more solid. Dealing with discrete space, time, and paths, refinements of the model may probably be achievable only with the help of computer simulations.

\section{Conflicts of Interest}

The author declares no conflicts of interest regarding the publication of this paper.

\section{References}

[1] Loll, R., Ambjorn, J. and Jurkiewicz, J. (2005) The Universe from Scratch. http://arXiv:hep-th/0509010

[2] Loll, R., Ambjorn J. and Jurkiewicz, J. (2005) Reconstructing the Universe. http://arXiv:hep-th/0505154

[3] Ambjorn, J., Jurkiewicz, J. and Loll, R. (2006) Quantum Gravity, or the Art of Building Spacetime. http://arXiv:hep-th/0604212

[4] Thiemann, T. (2007) Loop Quantum Gravity: An Inside View. Approaches to Fundamental Physics. Lecture Notes in Physics, 721, 185-263.

[5] Rovelli, C. (1998) Loop Quantum Gravity. Living Reviews in Relativity, 1, 1. http://www.livingreviews.org/lrr-1998-1

[6] Diel, H. (2016) Are Local Causal Models of Quantum Theory Feasible at All? arXiv:1604.03959v1

[7] Gambini, R. and Pullin, J. (2011) A First Course in Loop Quantum Gravity. Oxford University Press.

[8] Diel, H. (2016) Quantum Objects as Elementary Units of Causality and Locality. http://arXiv:1609.04242v1 
[9] Schutz, B.F. (2009) A First Course in General Relativity. Cambridge University Press, New York.

[10] Penrose, R. (2004) The Road to Reality. Vintage Books, New York.

[11] Baez, J.C. (1998) Spin Foam Models. http://arXiv:gr-qc/9709052

[12] Finster, F. and Kleiner, J. (2015) Causal Fermion Systems as a Candidate for a Unified Physical Theory. Journal of Physics: Conference Series, 626, 012020.

[13] Diel, H.H. (2017) A Model of Spacetime Dynamics with Embedded Quantum Objects. Reports in Advances of Physical Sciences, 1, 1750010.

[14] Mandl, F. and Shaw, G. (1993) Quantenfeldtheorie. AULA Verlag.

[15] Feynman, R.P. and Hibbs, A.R. (2010) Quantum Mechanics and Path Integrals. Dover Publication, New York.

[16] Diel, H.H. (2017) Collective Behavior in a Local Causal Model of Quantum Theory. Open Access Library Journal, 4, e3898. https://doi.org/10.4236/oalib.1103898

[17] Groessing, G. (2014) Emergence of Quantum Mechanics from a Sub-Quantum Statistical Mechanics. International Journal of Modern Physics B, 28, Article ID: 1450179.

[18] Verlinde, E.P. (2017) Emergent Gravity and the Dark Universe. SciPost Physics, 2, 016. https://doi.org/10.21468/SciPostPhys.2.3.016

[19] Fahr, H.J. (1990) The Maxwellian Alternative to the Dark Matter Problem in Galaxies. Astronomy \& Astrophysics, 236, 86-94.

[20] Wikipedia on "Galaxy Rotation Curve".

[21] Turyshev, S. and Toth, V. (2010) The Pioneer Anomaly. Living Reviews in Relativity, 13, 4. http://www.livingreviews.org/lrr-2010-4 https://doi.org/10.12942/lrr-2010-4

[22] Turyshev, S.G., Toth, V.T., Kinsella, G., Lee, S.-C., Lok, S.M. and Ellis, J. (2012) Support for the Thermal Origin of the Pioneer Anomaly. Physical Review Letters, 108, Article ID: 241101. https://doi.org/10.1103/PhysRevLett.108.241101

[23] Heighway, J.E. (2002) Toward a Deeper Understanding of General Relativity. 